\title{
Instrumento para el aprendizaje del movimiento armónico simple
}

DOI: $10.46932 / \mathrm{sfjdv2n1-061}$

Received in: November 1st, 2020

Accepted in: December 30th, 2020

\author{
Camilo Ernesto Pardo Beainy \\ Magister en Ingeniería Electrónica \\ Institución: Universidad Santo Tomás \\ Dirección completa: Av. Universitaria No. 45 - 202 Tunja - Colombia \\ Correo electrónico: camilo.pardo@usantoto.edu.co \\ Edgar Andres Gutierrez Caceres \\ Magister en Ingeniería Electrónica \\ Institución: Universidad Santo Tomás \\ Dirección completa: Av. Universitaria No. 45 - 202 Tunja - Colombia \\ Correo electrónico: edgar.gutierrez@ usantoto.edu.co \\ Luis Fredy Sosa Quintero \\ Magister en Ingeniería Electrónica \\ Institución: Universidad Santo Tomás \\ Dirección completa: Av. Universitaria No. 45 - 202 Tunja - Colombia \\ Correo electrónico: luis.sosa@usantoto.edu.co \\ Tania Carolina Barrera Molina \\ Ingeniera Electrónica \\ Institución: Universidad Santo Tomás \\ Dirección completa: Av. Universitaria No. 45 - 202 Tunja - Colombia \\ Correo electrónico: tania.barrera@usantoto.edu.co

\section{Oscar Fernando Camargo Amezquita} \\ Ingeniero Electrónico \\ Institución: Universidad Santo Tomás \\ Dirección completa: Av. Universitaria No. 45 - 202 Tunja - Colombia \\ Correo electrónico: oscar.camargo@usantoto.edu.co
}

\section{RESUMEN}

La enseñanza de la física en las instituciones educativas, es una de las que más evidencia mortalidad y sentimientos de dificultad en los estudiantes, es considerada como una asignatura teórico práctica, pero afronta la dificultad de que los ejercicios realizados en los laboratorios, pueden ser repetitivos en los diferentes niveles de formación y no se han incorporados los diferentes desarrollos tecnológicos que se ofrecen la electrónica y en general con las TIC.

Los estudiantes de los primeros semestres de los programas de ingeniería deben cursar la física mecánica, donde estudian la cinemática de un cuerpo, se desarrollan prácticas de laboratorio del movimiento uniforme, uniformemente acelerado, con dispositivos móviles, que pueden tener algunos elementos tecnológicos como, sensores de fin de curso y en el mejor de los casos, tomar lectura de tiempo, pero en muy pocas ocasiones pueden determinar la variables propias del fenómeno, como es la velocidad y la aceleración en sus diferentes categorías.

Hoy en día el desarrollo de la electrónica, permite desarrollar instrumentos que hagan tareas específicas en la medición de variables en unidades de tiempo que pueden ser más peñas que los microsegundos, en diferentes planos X Y Z de manera independiente, y con la ayuda de la ofimática se pueden unir para 
determinar el comportamiento de las variables en diferentes dimensiones y de esta forma dar una mayor profundidad al fenómeno analizado

Debido a estas razones, se ha diseñado un instrumento que permita medir las variables del Movimiento Armónico Simple, el dispositivo tienen la potencialidad de registrar al usuario y almacenar los datos obtenidos, para que sean procesados y analizados por el estudiante en unidades menores a la milésimas de segundo en las dimensiones que se le pidan. De esta forma se espera poder generar un aprendizaje más significativo y disminuir la mortalidad en esta área del conocimiento.

\section{INTRODUCCIÓN}

"Este apartado debe incluir de manera general uno o varios de los siguientes aspectos: Intención del autor, tesis o hipótesis de trabajo, el planteamiento del problema, información sobre los antecedentes y metas, objetivos y tipo de investigación” (Sánchez y Sánchez, 2010, p. 7).

En el año 1583, a la edad de 19 años, cuando Galileo asistía a una misa en el Domo observo el balanceo de una lámpara de aceite que colgaba del techo mediante un largo cable conocida como botafumeiro. Cuando el botafumeiro comenzó a oscilar, se movía rápidamente generando grandes arcos. Más tarde, cuando la oscilación había disminuido y el arco que describía era más pequeño la lámpara iba más despacio, pero el tiempo total de cada oscilación completa era siempre exactamente el mismo. La medida de tiempo empleada fue las pulsaciones que se tenían y de esta forma poder asegurar el que cada oscilación tenía lugar en el mismo periodo de tiempo (Zapata Jimenez \& Borja, 2014, pág. 2).

Esta observación género en Galileo, gran curiosidad de tal forma que una vez que se encontraba en su taller, procedió a repetir el fenómeno empleando bolas de plomo atadas a hilos. Su primera conclusión no tardo en parecer: sin importar el peso que estuviera sujeto al péndulo, el tiempo que necesitaba esta para completar su viaje ida y vuelta, era el mismo siempre y cuando la longitud de la cuerda fuera la misma. Como una segunda conclusión de la observación y repetición del fenómeno, le permitió deducir que aquello que cambiaba el tiempo de oscilación, era la variación en las longitudes de la cuerda que sostenían los pesos de plomo.

Esta observación condujo al invento del péndulo, el cual es usado hoy en diferentes a proceso de la industria, algunas veces de manera directa y otras indirectas, por ejemplo, en los relojes y otros instrumentos para medir con precisión el tiempo. Con el pasar de los siglo a esta observación y experimentación es lo que se conoce como Movimiento Armónico Simple "MAS” (Zapata Jimenez \& Borja, 2014, pág. 2).

\section{LOS CONCEPTOS}

El siglo XX es considerado, por muchos, como la centuria de la ciencia, de la democracia, de la extensión de los derechos civiles, por los movimientos generados en la década de los sesenta y setenta, por las amenazas de los desarrollos tecno-científicos, generados por merced al domino de la energía nuclear, la manipulación y control de las cargas eléctricas estáticas como dinámicas y por las interacciones que estos 
desarrollos tenían sobre la sociedad.

Estos movimientos se centraron en dos líneas que empezaron a marcar una ruta para lo que se ha conocido como Ciencia Tecnología y Sociedad; la primera de ellas se preocupa por los orígenes epistémicos y sociales, cultivada principalmente en Europa, en la Universidad de Edimburgo. La segunda, se centró en las consecuencias que ha tenido el desarrollo de este conocimiento en los diferentes espacios de la sociedad; se generaron así, las primeras oficinas de evaluación tecnológica y la implementación de políticas públicas en Ciencia y Tecnología, en los Estados Unidos de América (González, 1996).

Hoy, se puede considerar que los estudios de ciencia y tecnología se concentran en tres campos (González, 1996; Waks, 1990) a lo largo del mundo:

La investigación, que promueve una visión socialmente contextualizada de la ciencia y la tecnología.

Las políticas de ciencia y tecnología, que defienden la participación pública en la toma de decisiones y la gestión científico-tecnológica.

El educativo, tanto en la educación secundaria como universitaria, contribuye con una nueva y más amplia percepción de la ciencia y la tecnología, con el propósito de formar una ciudadanía alfabetizada científica y tecnológicamente.

Los ejes integradores de estos tres campos han sido la historia, la filosofía, la sociología de la ciencia y la tecnología, así como la economía del cambio técnico y las teorías de la educación y el pensamiento político, las cuales se han orientado hacia un trabajo de carácter interdisciplinario que busca promover y desarrollar nuevas formas de interpretación del fenómeno tecno científico.

Es en el marco educativo en donde se desarrollará este trabajo, ya que la influencia de la electricidad y especialmente el uso del electromagnetismo, ha cambiado de manera notable el mundo de hoy, y todo indica que generará cambios más grandes, para los cuales se debe estar preparados, no solo los docentes, sino la sociedad en general, ya que el uso de la tecnología ha superado los campos disciplinares específicos y se ha posicionado en cada una de las actividades humanas.

Ahora no es suficiente hablar de educación tecno-científica; se requiere incluir este término en los procesos de formación y selección de personal en todas las actividades humanas, convirtiéndose en una competencia fundamental para todo profesional, y para ello es necesario abordar la didáctica científica; más exactamente la trasposición de saberes, que permitirá no sólo analizar el carácter social de la ciencia y la tecnología, sino posibilitar espacios de reflexión sobre los modelos de desarrollo, la inequidad, el acceso a los nuevos bienes y servicios generados e incorporar sentido de responsabilidad, para poder encontrar la emancipación que la educación debe generar.

Una nueva esfera "El Sujeto en la Ciencia y la Tecnología", en las dificultades del aprendizaje de la física en los ingenieros, ya que hasta el momento, esos son los programas dedicados a educar y formar a los profesionales que se dedican y dedicarán al desarrollo, usos e innovaciones provocadas por la interpretación 
de la naturaleza, las cuales seguirán propendiendo hacia nuevos cambios que continuaran teniendo influencias, cada vez más impactantes, en todos los entornos sociales.

El currículo, los docentes y estudiantes de esta área del conocimiento deben fortalecer aún más las relaciones que tiene la historia, la historicidad, la responsabilidad e impactos de los desarrollos propios de su disciplina, para obtener una emancipación verdadera en el campo tecnológico y permitir el buen vivir de las sociedades con los beneficios generados por el desarrollo tecnológico y científico, en el cual estamos inmersos.

Lo anterior no solo es cumplir con los planteamientos y lecturas que se le hacen a la Ciencia, la Tecnología y la Sociedad, es responder al nuevo roll que tiene el docente dentro del aula de clase, buscando superar los modelos instruccionales, que han generado respuestas homogeneizadoras, con las cuales se desvirtúan el alcance tecno-científico y crean falsas expectativas de la calidad de vida y a su vez dan un valor más preponderante al poder existente, provocando desigualdades sociales y todos los males que ella conlleva.

El Movimiento Armónico Simple MAS, es definido como un movimiento que es periódico y es producido por una fuerza recuperadora. "El movimiento de la masa obedece a una ley representada por una ley sinusoidal” (Ramirez S. \& Mauricio, 1989, pág. 4). Este sistema presenta un movimiento oscilatorio y se considera que sobre este no hay fuerzas de rozamiento, evitándose así a disipación de energía y permitiéndose de este modo que "el movimiento se mantenga invariable, sin necesidad de comunicarle energía exterior a este" (Zapata Jimenez \& Borja, 2014, pág. 3)

La aceleración del M.A.S, es producida por una fuerza recuperadora, es decir, una fuerza que es proporcional al desplazamiento del móvil y va dirigida hacia el punto de equilibrio. Si es así, al sistema que oscila se le llama oscilador armónico, y es un modelo matemático que pocos osciladores reales cumplirán exactamente excepto en márgenes muy limitados (Ondas, pág. 121)

"El péndulo simple es una partícula de masa "m" suspendida de un punto "O" por una cuerda de longitud “ $L$ ”, que se puede considerar inextensible y de masa despreciable. A la partícula que oscila se le llama masa del péndulo" (Laura, 2013, pág. 1). Si se desplaza la partícula un determinado ángulo respecto a la posición de equilibrio, y al soltarla esta se moverá en un arco de circunferencia de radio " $L$ " del punto de referencia en el que se encuentra la partícula cuando está en equilibrio.

La amplitud " $A$ " será igual a la mitad de la longitud del arco que describe en su movimiento (igual a la distancia, medida sobre el arco, desde el punto de equilibrio a la posición de máxima separación), y la elongación "x" en cada momento será la distancia, medida sobre la trayectoria, desde el punto de referencia al punto en el que se encuentra en ese momento la masa del péndulo (Ondas, pág. 129). Todas estas características pueden ser visualizadas en la Figura 1. 
Fig.1 Pendulo simple con sus propiedades. Fuente: Ondas. EL PENDULO SIMPLE: un ejemplo de MAS.

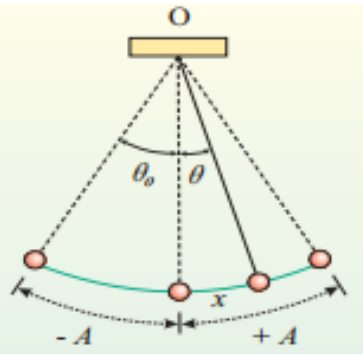

Recuperado de: http://docplayer.es/146168-El-movimiento-armonico-simple.html\#show_full_text

Sobre la masa actúan dos fuerzas en cualquier punto de la trayectoria: la atracción de la tierra sobre la masa cuyo valor es "mg” y la fuerza que ejerce la cuerda sobre la masa del péndulo " $T$ ”, lo cual se puede observar en la figura 2. Cabe aclarar que la tensión " $T$ " y la fuerza $n$ " $F_{n}$ " tienen la misma dirección pero sentido opuesto.

Fig.2 Fuerzas que influyen en el pendulo.

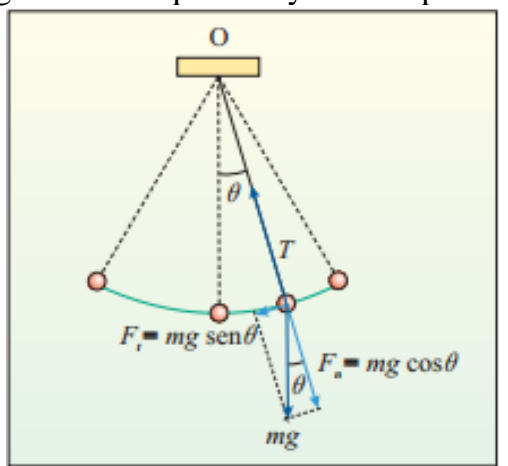

Fuente: Ondas. EL PENDULO SIMPLE: un ejemplo de MAS. Recuperado de: http://docplayer.es/146168-El-movimientoarmonico-simple.html\#show_full_text

La fuerza tangencial Ft produce una aceleración tangencial haciéndole cambiar su rapidez continuamente. Si se tiene en cuenta la relación que existe entre el ángulo expresado en radianes, el arco y el radio $(x=L \theta)$, y que para ángulos pequeños el seno del ángulo es aproximadamente igual al valor del ángulo expresado en radianes (lo cual se puede observar en la Tabla 1), se llega a la ecuación [1]:

\begin{tabular}{ccc} 
Tabla.1 Para $\theta<15^{\circ}$ la diferencia entre $\operatorname{sen} \theta$ y $\theta$ es menor d \\
\cline { 2 - 3 } $\boldsymbol{\theta}$ grados & $\boldsymbol{\theta}$ rad & $\boldsymbol{\theta}$ \\
\hline África & 564 & 68 \\
América & 9.541 & 6.521 \\
Asia & 8.451 & 2.254 \\
Europa & 9.154 & 2.521 \\
Oceanía & 1.684 & 354
\end{tabular}

Fuente: Ondas. EL PENDULO SIMPLE: un ejemplo de MAS. Recuperado de: http://docplayer.es/146168-El-movimientoarmonico-simple.html\#show_full_text 


$$
\begin{aligned}
\mathrm{Ft}=-\mathrm{mgsen} \theta & \approx-\mathrm{mg} \theta=-\mathrm{mg} \frac{\mathrm{x}}{\mathrm{L}}=-\frac{\mathrm{mg}}{\mathrm{L}} \mathrm{x} \\
& =-\mathrm{Kx}
\end{aligned}
$$

Donde k es una constante, cociente entre el peso de la masa y la longitud del péndulo.

El signo menos indica que el sentido de la fuerza es contrario al desplazamiento, tanto angular como lineal. La fuerza que produce la variación de la rapidez es proporcional a la distancia a la posición de equilibrio y de sentido contrario al desplazamiento, por lo que es de suponer que el movimiento del péndulo sea también un movimiento armónico simple, similar al de un cuerpo que se encuentra sujeto al extremo de un muelle (Ondas, pág. 129).

De este modo, se puede describir el movimiento del péndulo con la ecuación general del MAS, permite calcular la posición en función del tiempo, representada en la ecuación [2]:

$$
\mathrm{x}=\operatorname{Asen}\left(\omega \mathrm{t}+\phi_{0}\right) \quad \text { [2] Posición del pendulo en función del tiempo (x) }
$$

Hay que tener en cuenta que, si el ángulo no es pequeño, no se puede llegar a la misma aproximación, viéndose de este modo que sería un movimiento oscilatorio periódico, pero no se trataría del MAS, pues se dependería para este caso de la amplitud. Es así como se determina que un péndulo simple se comporta como oscilador armónico cuando tiene amplitudes pequeñas de oscilación, siendo así la fuerza recuperadora la componente tangencial del peso $\left(\mathrm{P}_{\mathrm{t}}\right)$ y la aceleración del péndulo es proporcional al desplazamiento, como se muestra en la ecuación [3].

$$
\mathrm{a}=-\frac{\mathrm{g}}{\mathrm{l} \cdot \mathrm{x}} \quad[3] \quad \text { Aceleración del pendulo }
$$

Dónde:

- $\quad$ : Aceleración del péndulo. Depende de la distancia a la posición de equilibrio x. Su unidad de medida en el Sistema Internacional es el metro por segundo al cuadrado $\left(\mathrm{m} / \mathrm{s}^{2}\right)$

- g: Aceleración de la gravedad. Su valor aproximado es $9.8 \mathrm{~m} / \mathrm{s}^{2}$

- $\quad$ 1: Longitud del péndulo. Su unidad de medida en el Sistema Internacional es el metro (m)

- x: Separación x de la vertical de equilibrio del péndulo. Su unidad de medida en el Sistema Internacional es el metro.

Cuando el péndulo se encuentra en reposo, en vertical, permanece en equilibrio ya que la fuerza peso es contrarrestada por la tensión en la cuerda (Ondas, pág. 129) 
Cuando la función seno es máxima, la función coseno es mínima, por lo cual hay un desfase entre seno y coseno de $\frac{\pi}{2} \mathrm{rad}$. Si se representan las dos funciones, se puede ver que la velocidad tiene los valores máximos y mínimos un cuarto de periodo antes que $x$. Como un cuarto periodo corresponde a un cambio de fase de $90^{\circ}$, a veces se deduce que la velocidad adelanta a $x$ en $90^{\circ}$. Todo esto puede observarse en la figura 3 que se muestra a continuación, en donde se muestra la posición, velocidad y aceleración.

Fig.3 Graficas de posicion, velocidad y aceleracion,respectivamente del MAS.

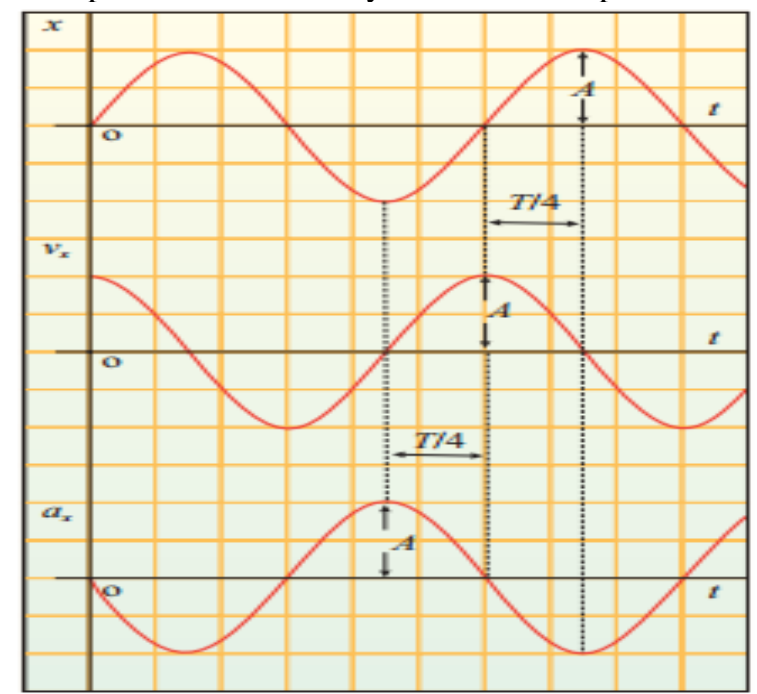

Fuente: Ondas. EL PENDULO SIMPLE: un ejemplo de MAS. Recuperado de: http://docplayer.es/146168-El-movimientoarmonico-simple.html\#show_full_text

La aceleración inmediata puede ser calculada mediante la ecuación [4]:

$$
\mathrm{a}=\frac{\mathrm{dv}}{\mathrm{dt}}=-\mathrm{A} \omega^{2} \operatorname{sen}\left(\omega \mathrm{t}+\phi_{0}\right)=-\omega^{2} \mathrm{x} \quad[4] \quad \text { Aceleración inmediata }
$$

La aceleración tiene un desfase con la elongación de $\pi \mathrm{rad}$, viéndose de este modo que las fases son iguales en valor absoluto, pero con diferente signo. El valor máximo de la aceleración es $A \omega^{2}$ (Ondas, pág. 124).

\section{METODOLOGÍA}

La investigación que se está desarrollando es de tipo exploratorio, donde la interpretación de los datos que se recolectan a través del protocolo de comunicaciones $\mathrm{I}^{2} \mathrm{C}$ permite develar el comportamiento del MAS. Para el desarrollo de la investigación se tuvieron en cuenta las siguientes fases:

Fase I: Establecer los conceptos y leyes física que intervienen en el Movimiento Amónico Simple. Con estos se establecen las variables que serán estudiadas y monitoreadas durante el proceso de la investigación. 
Fase II: Establecer la Instrumentación Electrónica y el modelo físico que se implementara para la medición y obtención de parámetros del Movimiento Armónico Simple, los cuales deben tener la potencialidad de tener una comunicación con diferentes medios de almacenamiento.

Fase III: Implementación del sistema teniendo en cuenta los diferentes tipos de obsolescencia y la modularidad que requieren los dispositivos en la actualidad.

Fase IV: Diseño e Interfaces con el usuario, donde debe responder a los criterios de usuarios de diferentes niveles de formación media, pregrado.

Fase V: Realizar pruebas con estudiantes para determinar el grado de facilidad de aprendizaje con el instrumento desarrollado vs estudiantes que no lo emplearon.

\section{RESULTADOS Y CONCLUSIONES}

En la actualidad se han podido determinar los conceptos fundamentales y leyes físicas que rigen el comportamiento del MAS, se han establecido las variables que se analizarán, conociendo los parámetros de entrada: longitud y la masa, el sistema es capaz de procesar el tiempo de recorrido (periodo), distancia recorrida, velocidad y con ellas se pueden procesar otras variables como son la aceleración instantánea, promedio y angular.

Se han determinado la instrumentación electrónica que se requiere para la implementación del sistema entre los que se encuentran:

El acelerómetro el cual tiene la potencialidad de medir en las tres dimensiones del espacio: X, Y, Z, generando una trama de datos, para cada una de las dimensiones que es comunicada a través del protocolo de comunicaciones $\mathrm{I}^{2} \mathrm{C}$. Para el análisis de este acelerómetro se tiene en cuenta la aceleración de la gravedad de la Tierra que es de aproximadamente $9.8 \mathrm{~m} / \mathrm{s}^{2}$. De este modo, la unidad de medición inercial (IMU) también detecta la aceleración de la gravedad terrestre, como se observa en la figura 4. "Gracias a la gravedad terrestre se pueden usar las lecturas del acelerómetro para saber cuál es el ángulo de inclinación respecto al eje X o eje Y" (Tr4nsduc7or, 2014). 
Fig.4 Funcionamiento del sensor con respecto a la aceleración de la gravedad.

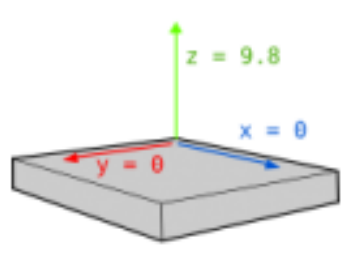

Paralelo al suelo

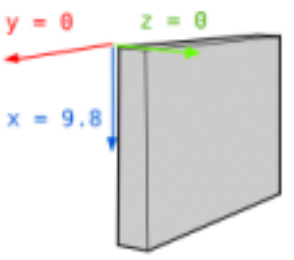

Girado 90 인

Fuente: Tr4nsduc7or. (2014). Tutorial de Arduino y MPU-6050. Recuperado de http://robologs.net/2014/10/15/tutorial-dearduino-y-mpu-6050/

Si la IMU está perfectamente alineada con el suelo, el eje Z marcará 9.8, y los otros dos ejes marcarán 0. Si giramos la IMU 90 grados. Ahora es el eje X el que está perpendicular al suelo, por lo tanto, marcará la aceleración de la gravedad. Si la gravedad es $9.8 \mathrm{~m} / \mathrm{s}^{2}$, y se podrá determinar la magnitud y demás magnitudes de los tres ejes del acelerómetro, por trigonometría es posible calcular el ángulo de inclinación de la IMU (Tr4nsduc7or, 2014).

Para calcular en ángulo se puede utilizar la ecuación [5a] y [5b] que aparecen a continuación:

$$
\begin{aligned}
& \text { AnguloY }=\arctan \left(\frac{\mathrm{x}}{\sqrt{\mathrm{y}^{2}+\mathrm{z}^{2}}}\right) \quad \text { [5a]Angulo en } \mathrm{Y} \\
& \text { AnguloX }=\arctan \left(\frac{\mathrm{y}}{\sqrt{\mathrm{x}^{2}+\mathrm{z}^{2}}}\right) \quad \text { [5b]Angulo en } \mathrm{X}
\end{aligned}
$$

Dado que el ángulo se calcula a partir de la gravedad, no es posible calcular el ángulo Z con esta ecuación [5] ni con ninguna otra. Para hacerlo se necesita otro componente: el magnetómetro, que es un tipo de brújula digital.

Para la medición de la distancia se empleó el principio del efecto Doppler, aplicado a instrumentos que funcionan por ultrasonido, a frecuencias superiores a los $20 \mathrm{KHz}$. La aplicación de estos dispositivos es como medidores de distancia, se puede apreciar en la figura 5 donde se tiene un emisor que emite un pulso ultrasónico, el cual rebota en un determinado objeto y la reflexión del puso es detectada por el receptor. 
Fig.5 Funcionamiento del sensor Utrasonico. Fuente: Los Autores

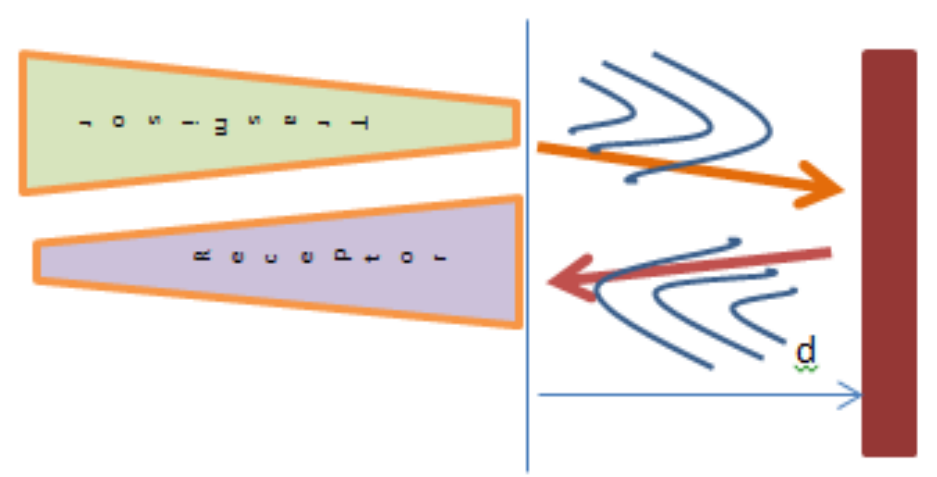

La mayoría de los sensores de ultrasonido económicos basan su funcionamiento en la emisión de un pulso ultrasónico cuyo campo de acción es de forma cónica. Midiendo el tiempo que trascurre entre la emisión y la percepción del eco se puede establecer la distancia a la que se encuentra el obstáculo que ha producido la reflexión, el modelo matemático conque se puede determinar se encuentra en la ecuación [6], donde $\mathrm{V}$ representa la velocidad del sonido en el aire y t el tiempo trascurrido entre la emisión y la recepción del pulso.

$$
d=\frac{1}{2} V \cdot t
$$

6 Determinación de la Distancia por Ultrasonido

Es importante resaltar que son diversos las problemáticas a superar con el manejo de esta tecnología, ya que se encuentran los fenómenos de la reflexión de las ondas por lo que una onda de ultrasonido tiene el mismo ángulo de incidencia y reflexión respecto a la normal de la superficie. Esto implica que si la orientación relativa de la superficie reflectora con respecto al eje del sensor de ultrasonido es mayor que un cierto umbral, el sensor nunca reciba el pulso de sonido que emitió. Si se intenta solucionar esta dificultad con una red de sensores se hace necesario hacer estudios para evitar los falsos ecos, situación que implica el manejo de tecnología de elevados costos.

En el efecto Hall, se hace presente cuando por una lámina conductora o semiconductora, se hace circular una corriente y se coloca en presencia de un campo magnético. Las cargas que están circulando experimentan una fuerza magnética $\vec{F}=q(\vec{v} \times \vec{B})$ y son desplazadas hacia uno de los bordes de la lámina. Esto hace que aparezca un exceso de carga negativa en uno de los bordes en tanto que en el otro aparece un exceso de carga positiva, lo que provoca que aparezca un campo eléctrico E, que a su vez ejerce una fuerza de carácter eléctrico sobre las cargas $\vec{F}=q \vec{E}$. La acumulación de cargas continua hasta que el campo eléctrico se hace suficientemente grande como para que la fuerza eléctrica compense a la magnética. Esta situación se caracteriza por la diferencia de potencial que aparece entre los bordes denominada voltaje Hall, si I es la intensidad de corriente, B el campo magnético, $\mathrm{n}$ la densidad de portadores, q su carga y del ancho 
de la lámina, el potencial se puede modelar como:

$$
V_{\text {Hall }}=\frac{I B}{n q d}
$$

Con este principio se colocan las condiciones iniciales del sistema, es decir se coloca un punto de referencia de la masa del péndulo, y de esta forma se puede determinar no solo la distancia de la cuerda del péndulo, sino la posición y con ellas se determinará el ángulo de inicio del péndulo, variables fundamentales para la comprensión y la interpretación del Movimiento Armónico Simple

Para la Interfaz Usuario Humano, se ha trabajado una matriz Pantalla de Cristal Líquido graficadora (GLCD), en la cual se puede colocar un logo institucional o un nombre, y generar parámetros de administración para tener control de quien emplea y de esta manera poder desarrollar practicas personalizadas que puedan generar reportes. En la figura 6, se aprecia una borrador de cómo se puede identificar el instrumento diseñado.

Fig.6. GLCD con presentacion inicial del dispsotivo para realizar practicas de Movimiento Armonico Simple.

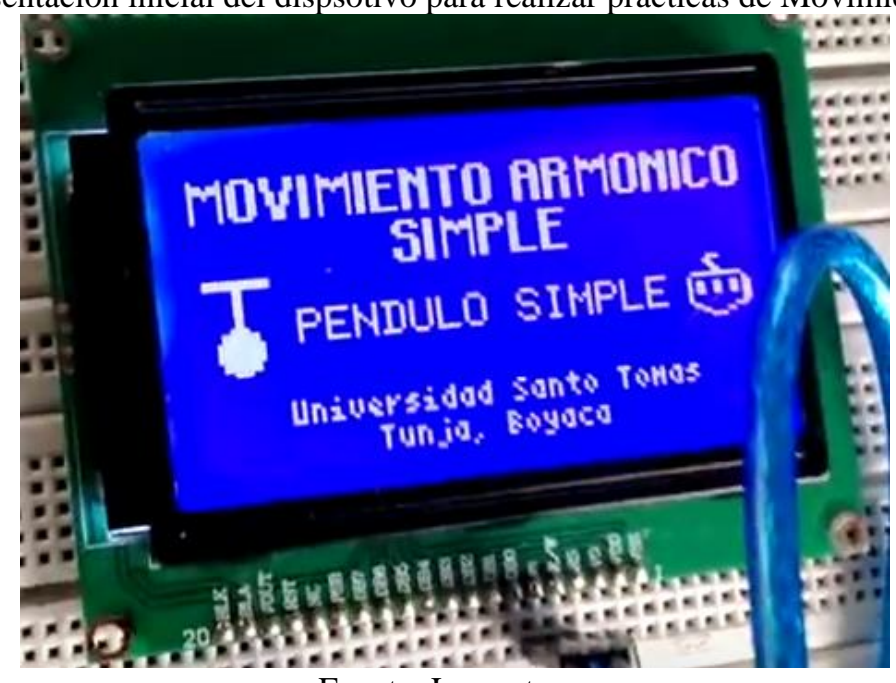

Fuente: Los autores 


\section{REFERENCIAS}

González. G; Matajira, L; \& Rivera, G. (1996): Ciencia, tecnología y sociedad, una introducción al estudio social de la ciencia y la tecnología. Madrid, Tecnos.

Waks, L., y Rostum, R. (1990). El ABC de ciencia, tecnología y sociedad, en: National STS Net-Work, Pennsylvania State University.

Jiang, X., Bi, T., Wang, Y., \& Ouyang, J. (2011). A Simple Pendulum Experiment for Energy Compensation Automation, 7142-7145.

Engineering, P. (2011). Available online at www.sciencedirect.com Open access under CC BY-NC-ND license., 10, 3734-3740. doi: 10.1016/j.proeng.2011.04.611

Bermúdez, H. H., B, H. A. G., \& Bermúdez, H. F. (2011). Prototipo mecatrónico para la enseñanza y el aprendizaje del movimiento armónico simple Mechatronics prototype for teaching and learning the simple harmonic motion, (49), 245-252.

Kavithaa, R., Babu, R. U., \& Deepak, C. R. (2013). Simple pendulum analysis - A vision based approach. 2013 4th International Conference on Computing, Communications and Networking Technologies, ICCCNT 2013, (Figure 1). doi:10.1109/ICCCNT.2013.6726567

Butterworth, J. (2014). Simple harmonic motion: the swing of the pendulum | Jon ... Guardian. Retrieved from https://www.theguardian.com/science/life-and-physics/2014/may/18/simple-harmonic-motion-theswing-of-the-pendulum

Hernández-Sampieri, R., Fernández-Collado, C., \& Baptista-Lucio, P. (2006). Análisis de los datos cuantitativos. Metodología de la investigación.

Historia de la Física. (n.d.). Retrieved from http://mural.uv.es/sansipun/

Las leyes del Pendulo Fisico Oscilacion Periodo Frecuencia. (n.d.). Retrieved from http://historiaybiografias.com/pendulo/

Movimiento Armonico Simple.pdf. $\quad$ (n.d.). $\quad$ Retrieved from http://www.proyectosalonhogar.com/enciclopedia_ilustrada/ciencias/Movimiento_Armonico2.htm

Serway, R. a., \& Kirkpatrick, L. D. (1988). Physics for Scientists and Engineers with Modern Physics. The Physics Teacher (Vol. 26). http://doi.org/10.1119/1.2342517

Vizca Lajaraíno, J. R., \& Pelegrí Sebastiá, J. (2007). LABVIEW Entorno gráfico de programación. In M. S.A (Ed.), LABVIEW Entorno gráfico de programación (p. 385). Retrieved from: http://site.ebrary.com.bdatos.usantotomas.edu.co:2048/lib/bibliotecaustasp/reader.action?docID=10204103 Instruments, N. (2016). ¿Qué es Adquisición de Datos? Daq. Retrieved from http://www.ni.com/dataacquisition/what-is/esa/

UCM. (100AD). Manual Básico de Programación en Labview (Vol. 1).

Holguín, G., Pérez, S., \& Orozco, Á. (2002). Curso Básico de LabVIEW 6i.

Ruiz Guti, M. (2007). Manual de Programación Arduino: Manual de Programación. Arduino Notebook, 1, $3-70$ 
Baeza, J. P., \& Pomares, J. (2009). Manual de arduino revision. Manual De Arduino, 1-8.

Diosdado, R. (2014). Manual Arduino. Saudi Med J, 33, 3-8. http://doi.org/10.1073/pnas.0703993104

Ibraim Hernández, Juan Fajardo, Oskar Vanegas, J. B. (2011). Guía básica de arduino, 120.

Matamoros S, A. Z. (2009). Ejemplo de un Péndulo Simple. Mérida, Venezuela. Retrieved from http://www.webdelprofesor.ula.ve/ingenieria/matamoros/index_archivos/DF_SNL.pdf 